\title{
Estrategias de modernización empresarial: Procesos, productos y fuerza de trabajo*
}

\author{
Gamboa Cáceres, Teresa** \\ Arellano Rodríguez, Madelein \\ Nava Vásquez, Yuneska \\ ** Investigadoras del Centro de Estudios de la Empresa, FCES, Universidad del Zulia. \\ Maracaibo, Venezuela. E-mail: teresagamboa@cantv.net; marellano@luz.ve; \\ yuneskanava@hotmail.com
}

\section{Resumen}

Con base en la conceptualización general de las estrategias empresariales elaborada en un trabajo previo, se realiza la revisión y discusión de bibliografía y experiencias acerca de aquellas estrategias que tienen como propósito fortalecer las funciones esenciales de la empresa. Las estrategias intraempresa se clasifican tomando como criterio los elementos que intervienen en la producción desde una perspectiva sistémica, encontrando tres tipos: modernización de procesos, estrategias sobre la fuerza de trabajo y estrategias relativas al producto, cada uno de los cuales tiene subtipos particulares. Se concluye que las estrategias intraempresa centran la atención en fines relacionados con la reducción de costos y con la competencia, especialmente para adquirir mayor capacidad operativa, a fin de responder a las fluctuaciones del mercado, evitar su saturación y ampliar la participación en el mismo; en síntesis, para obtener ventajas comparativas frente a los competidores. Sin embargo, algunas de las estrategias intraempresa, si bien reducen los costos y mejoran la posición competitiva en el mercado, provocan desempleo y precarizan las condiciones de trabajo; pero además, la generalización del desempleo reduce la capacidad adquisitiva del mercado. Se destaca además, la necesidad de abordar la innovación y modernización considerando las características y necesidades del contexto.

Palabras clave: Modernización tecnológica, modernización informática, sistemas flexibles de producción, contratación laboral, gestión industrial.

\section{Strategies for Business Modernization: Proceses, Products and Work Force}

\section{Abstract}

Based on a general conceptualization of management strategies elaborated in a previous 
study, a review and discussion of bibliography and experiences is undertaken in relation to strategies the purpose of which are to strengthen the essential functions of the company. Intra-business strategies are classified using criteria related to elements that intervene in the production process from a systemic perspective, where three types of elements are found: process modernization, labor strategies, and product strategies, each of which has very particular sub-categories. The conclusion is that the intra-business strategies center attention on fine relationships that reduce costs and with competition, especially in acquiring greater operative space in order to respond to market fluctuations, avoid saturation and increase market párticipation, provoking unemployment and precarious labor conditions and also generalizing reduced employment and market purchase capacity. The study also points out the need to touch on innovation and modernization considering contextual characteristics and needs.

Key words: Technological modernization, informational (informatics) modernization, flexible production systems, labor contracts, inducatrial management.

Recibido: 03-07-16. Aceptado: 03-09-17

\section{I ntroducción}

Partimos de la definición de estrategias empresariales desarrollada previamente, en la cual se conciben como: “... los medios para lograr cambios a mediano y largo plazo, con el propósito de obtener ventajas comparativas u oponerse a las actuaciones presentes o futuras de los competidores... A ellas subyacen principios, valores y fines que determinan la dirección de la empresa; además, la estrategia exige un conjunto de esfuerzos para coordinar e integrar en un todo coherente, recursos y operaciones en función de alcanzar fines u objetivos generales...". Previamente se realizó también una aproximación tipológica, tomando como criterio de clasificación la naturaleza de las relaciones entre los actores que intervienen, de lo cual surgieron las siguientes categorías generales de estrategias empresariales: de colaboración entre empresas; agresivas contra otras empresas, orientadas hacia los clientes para mantener o ampliar la participación en el mercado; aquellas que expresan vínculos empresa-gobierno; las que corresponden a la relación entre la empresa y el sindicato; y finalmente, las estrategias que implican interacciones dentro de la empresa (Gamboa y otras, 2001:138, 147).

La opinión de Villalba (1996:59-61) respecto a la potestad de cada organización para definir los ímites de acción de su negocio y establecer estrategias para optimizar sus procesos internos, no es absoluta; está condicionada por factores del entorno, tales como 
el grado de desarrollo y difusión de tecnologías (físicas y sociales), el acceso al crédito, las políticas gubernamentales, los vínculos con organizaciones más avanzadas y la capacidad de resistencia de los trabajadores, entre otros. También está relativamente condicionada por la responsabilidad social de la organización, en términos del desarrollo humano sustentable.

Ahora bien, los cambios generados por las nuevas estrategias intraempresa durante la segunda mitad del siglo $\mathrm{XX}$, desde el punto de vista técnico, organizativo y laboral, no constituyen la continuación del modelo tradicional de racionalización y tecnificación, al punto que Coriat (1995) destaca la necesidad de "pensar al revés".

Las nuevas estrategias se inscriben en la b́gica del capital y buscan: incrementar la productividad, reducir costos y tiempos de respuesta, 'mejorar' el producto o sustituirlo, etc.; todo ello para optimizar la posición competitiva en el mercado y, por lo tanto, la rentabilidad. En su afán por reducir costos operativos, las empresas incrementan la inversión de capital en un ambiente de presiones por la innovación y modernización tecnológica, y modifican sus procesos de trabajo adecuándolos a las nuevas tecnologías incorporadas, lesionando el factor más sensible, que es el trabajo. Sin embargo, también es posible (Shumacher, 1990; Semler, 1999) el desarrollo de propuestas con enfoques que, sin sacrificar el éxito empresarial, consideren la posibilidad de servir al hombre y al ambiente en lugar de destruirlos.

En esta ocasión centramos la atención en las estrategias intraempresa, en tanto ocurren al interior de la empresa y tienen como propósito general fortalecer las funciones esenciales del negocio. Su clasificación se realizó tomando en consideración los elementos que intervienen en la producción, desde una perspectiva sistémica, lo cual dio lugar a los siguientes tipos: a) modernización de los procesos donde se interrelacionan los factores de la producción (medios, objetos y fuerza laboral); b) estrategias sobre la fuerza de trabajo, que constituye el factor más sensible e indispensable; y c) estrategias relativas al producto resultante del proceso (Ver Cuadro 1). Las estrategias relacionadas con los objetos de trabajo (insumos) son fundamentalmente estrategias interempresas, de relación con los proveedores, que no serán discutidas en este trabajo.

Las estrategias intraempresa se inscriben en un nuevo modelo de acumulación de capital, acorde a las condiciones actuales de desarrollo de las fuerzas productivas, así como a las exigencias de los mercados. En este artículo se caracterizarán dichas estrategias, así como las consecuencias en los trabajadores y en la sociedad en su conjunto. 


\section{Modernización de procesos}

Los cambios organizativos, conjuntamente con el cambio tecnológico en la producción, son denominados por Dombois y Pries (1993:13) Modernización Industrial o Empresarial; según los autores se trata de un proceso de carácter sistémico que se inicia en Japón a mediados de los años cincuenta y se difunde con variantes en todo el mundo. Las nuevas tecnologías actúan como capital productivo para facilitar la producción de bienes y servicios diferenciados e incrementar la productividad del trabajo y por lo tanto, potenciar la valorización del capital.

Sin embargo, de acuerdo con Ermida (1999:17-32), a pesar de sus bondades en cuanto desarrollo tecnológico, comercio, producción, e información, este efecto globalizador contiene amenazas directas no sólo para los trabajadores, sino para la sociedad en general, porque lesiona las posibilidades de equidad social, como se apreciará en la discusión de las diversas estrategias.

\subsection{Modernización informática}

Según Albizu (1997:7-8) la informática y tecnologías afines han experimentado un desarrollo sin precedentes en la historia, caracterizado por: a) gran rapidez de sus innovaciones; b) universalidad de sus aplicaciones; c) facilidad de difusión; d) repercusión de las nuevas aplicaciones en el grupo tecnológico; e) sustitución progresiva de personas por máquinas; y f) cambios estructurales en las empresas. 


\section{Cuadro I}

Estrategias intraempresa para fortalecer las funciones esenciales

\begin{tabular}{|c|c|c|}
\hline Tipo & Estrategia Especifica & Fines \\
\hline \multirow{3}{*}{$\begin{array}{l}\text { Modernización } \\
\text { de Procesos }\end{array}$} & Modernización Informática & \multirow{3}{*}{$\begin{array}{l}\text { - Adquirir mayor capacidad para res- } \\
\text { ponder a fluctuaciones del mercado } \\
\text { - Reducir costos a mediano y largo } \\
\text { plazo } \\
\text { - Optimizar Procesos }\end{array}$} \\
\hline & $\begin{array}{l}\text { Modernización Tecnológica } \\
\text { en Producción }\end{array}$ & \\
\hline & Reestructuración Organizativa & \\
\hline \multirow{2}{*}{$\begin{array}{c}\text { Estrategias } \\
\text { sobre la Fuerza } \\
\text { de Trabajo }\end{array}$} & $\begin{array}{l}\text { Flexibilización del Contrato } \\
\text { de Trabajo }\end{array}$ & - Reducir costos \\
\hline & Socialización Laboral & $\begin{array}{l}\text { - Desarrollar identidad corporativa } \\
\text { - Control ideológico de la fuerza } \\
\text { de trabajo }\end{array}$ \\
\hline \multirow[t]{3}{*}{$\begin{array}{c}\text { Estrategias Relativas } \\
\text { al Producto }\end{array}$} & Desarrollo de Productos & $\begin{array}{l}\text { - Evitar la saturación del mercado } \\
\text { - Acelerar el desgaste del producto } \\
\text { - Adquirir ventajas frente a los } \\
\text { competidores }\end{array}$ \\
\hline & Diversificación Productiva & $\begin{array}{l}\text { - Evitar la dependencia de un solo } \\
\text { negocio } \\
\text { - Ampliar el mercado }\end{array}$ \\
\hline & Concentración Productiva & $\begin{array}{l}\text { - Fortalecer línea con mayor mercado } \\
\text { potencial } \\
\text { - Reducir costos } \\
\text { - Satisfacer clientes en mejores } \\
\text { condiciones }\end{array}$ \\
\hline
\end{tabular}

Fuente: Elaboración propia.

Tal como afirma Castells (1998: 75), "se ha constituido un sistema tecnológico, que se configura como nuevo paradigma operativo en la década de los setenta,... funcionando con reglas cada vez más homogéneas entre las empresas y territorios que lo constituyen". Dicho paradigma se basa en el uso de novedosas tecnologías de información e interconexión en red, aplicables en todos los sectores de la economía.

Los desarrollos informáticos y en telecomunicaciones exigen a las empresas usar creativamente los sistemas de información y de gestión; es posible centralizar la toma de decisiones porque se potencia enormemente la posibilidad de coordinación y control a lo largo y ancho de las diferentes estructuras de la organización -Intranet-, así como entre empresas y agencias gubernamentales de todo el mundo -Internet-.

Internet es una de las nuevas herramientas tecnológicas que incrementa el acceso a la información en todos los campos; es un sistema de comunicaciones digitales de alta velocidad, que puede manejar en tiempo real y en forma interactiva datos de computador, 
señales de televisión y de teléfono (Hasty y Reardon, 1998:583),... enormes cantidades de texto, sonido, imágenes y video, dentro y fuera de los hogares, negocios, fábricas, hospitales, centros educativos, y las oficinas gubernamentales; esta red pública e interconectada disponible para todos, es el medio que permite a los computadores del mundo comunicarse entre sí (Tapscott, 1997:12).

De manera similar, el comercio electrónico contribuye a la globalización creciente de la producción, la distribución y el consumo, ampliadas por el desarrollo en los sistemas de comunicación y transporte; esto requiere el establecimiento de instituciones y marcos jurídicos globales (Carreón, 2001:1) que aún no se han consolidado.

El objetivo empresarial de la modernización informática es obtener mayor productividad y acceso a los mercados; las nuevas tecnologías incrementan las posibilidades de control sobre el trabajador y sobre el ciudadano, aunque también permiten democratizar la información. Según Sierra (1999:6) “La propagación de redes mundiales de telecomunicaciones... representa la posibilidad, real, innegable por tanto, del acceso universal, casi ilimitado a la información y al conocimiento".

\subsection{Modernización tecnológica en la producción}

Actualmente las empresas tienen la posibilidad de usar los sistemas de producción informatizada, con la finalidad de coordinar actividades dispersas e incrementar la flexibilidad que les permita entrar y salir de diferentes mercados, alterar diseños de producción y controlar los comportamientos de su personal. Los sistemas de control numérico computarizado o programables por ordenador, permiten que las empresas optimicen la cadena de producción y los productos, reduciendo la infraestructura y los costes. Además, los ordenadores personales y la tecnología de información facilitan enormemente la coordinación internacional y el control de la producción y comercialización (Harrison, 1997:21-22).

La modernización tecnológica en producción es una estrategia empresarial que consiste en la innovación o la adopción de nueva tecnología en el proceso productivo (Olivera, 1997:8); en búsqueda de productividad, calidad y competitividad, la empresa incorpora cambios tecnológicos para responder más eficientemente a las necesidades de un mercado globalizado. La innovación o adopción de tecnologías duras, como la robotización y la automatización de los medios de trabajo, requieren cambios en la organización de los procesos, flexibilizar el trabajo y modificar el enfoque de producción en línea, modalidades que serán discutidas en la sección correspondiente a reestructuración. 
La decisión sobre cómo realizar la modernización dependerá de las presiones del mercado, la disponibilidad de capital y de las áreas prioritarias para la actividad específica de la empresa. En el caso venezolano, Sant Roz (2003:49-50) discute cómo a causa de la imposición tecnológica, Venezuela ha sido invadida durante décadas por maquinaria industrial compleja - no pocas veces "Ilave en mano" con técnicos incluidos-; la desactualización sistemática, a causa de la "voraz producción tecnológica de los países poderosos", y el rentismo petrolero, han generado: a) elevada capacidad ociosa; b) inundación de chatarra tecnológica; c) incremento de la deuda externa por el alto costo de la "repotenciación" o "reconversión" industrial; d) deterioro del sector productivo nacional, porque a cada invento tecnológico sigue la importación de "nuevos" productos de consumo final; y por último, e) un desarrollo científico tecnológico cada vez mas inalcanzable, si el modelo es el de los países del llamado primer mundo.

Por otra parte, Sotelo (1999:87) destaca que el progreso técnico ha llevado históricamente a una reducción sistemática del tiempo de trabajo vivo necesario, como contrapartida al incremento de la productividad por las nuevas tecnologías. Esto profundiza el desempleo, aumenta la brecha de desigualdad e inseguridad social y disminuye la calidad de vida de los ciudadanos, especialmente en América Latina que según Iglesias (1999: 14) presidente del Banco Interamericano de Desarrollo, es la región con mayor grado de inequidad en el mundo. Además, según Ramos (1997:1) la productividad en latinoamérica es sólo un tercio comparada con las empresas de países desarrollados y cerrar esta brecha requiere de la modernización tecnológica. Es decir, estamos en un círculo vicioso, que requiere imaginación y creatividad para romperlo.

Tal como afirma Shumacher (1990:135), es posible que la pobreza de los países del tercer mundo les impida adoptar tecnología importada, favoreciendo también el desempleo por falta de capacidad competitiva en un mundo globalizado, lo cual incrementa las tensiones sociales. De allí la necesidad de crear "una tecnología con rostro humano", que no sólo aumente la productividad, sino que además incite a "las personas a ser creativas y productivas en sí mismas... una tecnología intermedia, intensiva en mano de obra, útil para establecimientos de pequeña escala; en última instancia una tecnología que sirva al hombre en lugar de destruirlo".

\subsection{Reestructuración organizativa}

Las transformaciones del mercado después de la Segunda Guerra Mundial, con una demanda reducida de productos diferenciados, forzaron en la empresa japonesa Toyota, 
bajo la dirección del Ingeniero Ohno, el desarrollo de un modelo que según su creador se fundamenta en dos principios: la producción en el momento preciso (justo a tiempo) y la auto activación de la producción; ambos principios se expresan en diversas técnicas y formas de actuación perfectamente integradas, cuya aplicación aislada le resta fuerza al modelo (Coriat, 1995:13). El nuevo paradigma modifica totalmente el modelo fordtaylorista que respondía a mercados masivos y homogéneos.

A partir de los años ochenta, paralelo a la incorporación de nuevas tecnologías microelectrónicas, informáticas y telemáticas, se generaliza la reestructuración, reconversión o reingeniería del sistema de producción, primero en Estados Unidos y Europa; finalmente llega a Latinoamérica, aunque de una manera menos decidida y más selectiva. La reestructuración organizativa se fundamenta en la automatización de las operaciones para reducir costos y tiempos de espera; consiste en primer lugar en deshacerse de algunos procesos no medulares, rediseñar los procesos básicos del negocio y efectuar cambios en la dirección y control de la producción.

Un aspecto clave de la reestructuración organizativa consiste en transferir algunos procesos de apoyo, a trabajadores totalmente flexibilizados -cuya labor se controla mediante redes digitales-, o a proveedores de bienes y servicios (outsourcing 0 tercerización); esto conduce a todo un conjunto de estrategias interempresas, de integración vertical con los suplidores (que serán discutidas en otro trabajo), las cuales facilitan la difusión de las nuevas formas organizativas entre las empresas integradas.

La modificación de los procesos básicos requiere: a) distribución de hombres y máquinas (lay-out) para dar mayor fluidez al proceso productivo y facilitar que varios equipos o tareas sean atendidos por un trabajador polivalente (integración de funciones y tareas); b) Producción en línea con tareas compartibles en celdas de trabajo, que hacen al proceso productivo más flexible (Coriat, 1995:57; Olivera, 1997:8). Se introducen así características del trabajo que fueron originalmente objetivos de la lucha sindical, como la polivalencia y el trabajo integral, pero al precio de la intensificación del trabajo, su precarización y la reducción de la oferta de empleo.

La integración de funciones y tareas compartibles entre miembros del equipo, incorpora la posibilidad de que los trabajadores tomen ciertas decisiones operativas y se autocontrolen (empowerment). No obstante, hay experiencias empresariales más avanzadas pero aún de difícil generalización (Semler, 1999:12, 169, 197, 288), que incluyen por ejemplo, decisiones relativas a: contratar y despedir al jefe, nuevas inversiones, ubicación y distribución de la fábrica, hora de entrada y salida del trabajo, 
metas de producción, participación en los beneficios; esto requiere acceso a la información contable y estadística, históricamente consideradas secreto mercantil.

Los cambios organizativos generan un achatamiento de la estructura piramidal; la automatización, la intensificación del trabajo, el empoderamiento y la subcontratación, implican la reducción significativa de los trabajadores de base, así como los niveles medios de supervisión y gerencia, conformando la "fábrica delgada" de Ohno y lo que otros autores como Woolf (1995) Ilaman despreocupadamente, la "pérdida de grasa" (es decir, la reducción de trabajadores).

En síntesis, la reestructuración organizativa da lugar a un nuevo paradigma, que modifica aspectos como la distribución de tareas y responsabilidades, la duración de la jornada, la estabilidad laboral, el sitio de trabajo, el sistema de remuneración, la capacitación y las relaciones empresa-sindicato. En definitiva, conduce a la flexibilización de las relaciones laborales.

\section{Estrategias sobre la fuerza de trabajo}

La competencia internacional presiona a los empresarios para utilizar las nuevas tecnologías en el proceso productivo; pero estos cambios exigen modalidades flexibles de relación con los trabajadores y con otras empresas. La flexibilidad de las relaciones laborales mejora la capacidad de respuesta a las fluctuaciones del mercado, al permitir variaciones cuantitativas y cualitativas de la fuerza de trabajo. Sin embargo, colateralmente la inestabilidad laboral afecta en forma negativa la socialización de los valores corporativos y por lo tanto, el compromiso de los trabajadores con la empresa; igualmente lesiona las políticas internas de capacitación laboral. En las siguientes secciones discutiremos las estrategias flexibilizadoras y las de socialización, que expresan la relación de la empresa con un factor de producción indispensable: la fuerza de trabajo.

\subsection{Flexibilización del contrato de trabajo}

La automatización y la reestructuración radical de la organización interna de las empresas exige ajustar el número de trabajadores a las fluctuaciones de la demanda; esto conduce necesariamente a modificar los contratos y las condiciones de trabajo, lo cual provoca fuertes reacciones de los sindicatos, por los despidos masivos y la intensificación del trabajo. Vale recordar que en la empresa Toyota (Coriat, 1995:35-37), promotora de los cambios organizativos, sólo se consolidó el modelo japonés tras sucesivas derrotas sindicales, hasta convertir a la organización laboral en un "sindicato de empresa", al punto 
que para ser jefe de personal se requiere haber sido presidente del sindicato; pero en contrapartida los japoneses crearon mercados laborales internos de por vida para los trabajadores de los procesos básicos.

La generalización del nuevo modelo de acumulación de capital, ha venido transformando el sistema de relaciones laborales, conquistado gracias a las luchas sindicales y a la correlación internacional de fuerzas, que forzaron concesiones laborales. Las conquistas se habían consagrado en las respectivas legislaciones del trabajo, pero surgen presiones para modificarlas (desregulación), especialmente aquellas relativas a la estabilidad, definición del cargo y beneficios laborales.

El sistema de relaciones laborales con cierto grado de protección al trabajador no alcanzó a sobrevivir un siglo; nació y murió durante el siglo XX y costó vidas de los trabajadores en diversos lugares del mundo. Este sistema, que ya parecía "tradicional" tuvo como rasgos fundamentales los siguientes (Suárez y otros, 2001): a) estabilidad en el trabajo por tiempo indeterminado con el mismo empleador; b) contenido muy preciso del puesto de trabajo, con tareas y responsabilidades específicas; c) remuneración fija; d) jornada completa, estipulada legalmente y con pago del tiempo extra laborado; y e) trabajo dentro de la empresa.

Los cambios en curso son tan radicales que algunos autores hablan de "el fin del trabajo" (Rifkin, 1996) o con más precisión, de "el fin del empleo" (Bridges, 1994:14-21), dado que se reducen las oportunidades de trabajo asalariado; numerosos empleos se convierten en "situaciones laborales" bajo contrato temporal y a destajo según productividad, preferiblemente con un trabajador o grupo convertido en organización (microempresa, cooperativa u organización no gubernamental -ONG); eventualmente el trabajo debe ser realizado fuera de los muros de la empresa. Tales contratos no permiten sobrevivir con una sola oportunidad laboral y por lo tanto se impone la necesidad de tener varios "patronos".

En estas circunstancias se institucionalizan diversas formas de flexibilidad laboral: de la duración del contrato, del salario, de la jornada, del lugar de trabajo, a las cuales subyace la eliminación de la estabilidad y la reducción de costos laborales. Los trabajadores contratados bajo modalidades flexibles constituyen una categoría especial, periférica, sin seguridad social. Sólo un pequeño grupo de trabajadores correspondiente al núcleo del negocio (procesos básicos) conservan la estabilidad y ciertas condiciones de la seguridad social del modelo tradicional. 
En un contexto de flexibilización, el rol del Estado en la regulación y mediación de las relaciones capital/trabajo, se ve disminuido. De igual forma las organizaciones sindicales pierden progresivamente capacidad de lucha y de negociación, sin que el Estado ni los sindicatos hayan logrado definir posibles líneas de acción y organización (Montero y otros, 1999: 11).

Sotelo (1999:100) considera que lo esencial del nuevo modelo de acumulación es la flexibilidad del trabajo y la desregulación de las relaciones laborales, que profundizan la precarización y la inseguridad en el trabajo; lo anterior es una consecuencia de la reestructuración del sistema capitalista, que implica un "impresionante despliegue de fuerzas en la innovación tecnológica y su aplicación a los procesos productivos".

\subsection{Socialización laboral}

El paso de los modelos autoritarios de dirección al sistema de relaciones humanas, puso sobre el tapete la importancia para la productividad, de factores como la motivación, madurez, posibilidad de innovación y satisfacción del individuo; en consecuencia, se crearon oportunidades para ampliar la concepción de capacitación más allá del adiestramiento para el desempeño de tareas específicas. Surge así la socialización como una estrategia empresarial.

La socialización laboral es el proceso por el cual un trabajador aprende los valores, normas, deberes y derechos, formas de expresión y relación, predisposiciones, capacidades y motivaciones, que configuran la identificación y compromiso con los intereses de la empresa, e inducen los modelos de comportamiento esperados por esta.

En general, la socialización laboral puede ser primaria cuando un individuo inicia su vida laboral y secundaria, cuando cambia de empleo, de rol o de nivel. I gualmente pueden ser necesarios procesos de resocialización, cuando una organización intenta impulsar cambios políticos o de modernización.

Ahora bien, en una organización sujeta a flexibilidad laboral, hay una rotación permanente de trabajadores, que afecta las posibilidades de socialización y por lo tanto lesiona el compromiso del personal con la institución. Sin embargo, las organizaciones no pueden renunciar totalmente a los trabajadores permanentes, necesarios para la realización de los procesos básicos (core bussines); éstos disfrutan de estabilidad relativa con ciertos beneficios sociales y constituyen el núcleo de la organización (Albizu, 1997:20); con el fin de conservar su estabilidad, renuncian implícitamente a algunos 
derechos laborales legales y contractuales, pero la empresa tiene la posibilidad de desarrollar en ellos identidad corporativa, mediante diferentes mecanismos socializadores, tales como: la inducción, talleres de crecimiento personal, divulgación de un periódico o revista institucional, clubes empresariales, concursos y premios, etc.

Por el contrario, los trabajadores periféricos, sujetos a rotación, tienden a perder la identificación con una organización que los toma o los deja de acuerdo a las fluctuaciones de la demanda. Esto plantea a la empresa la necesidad de revisar sus criterios de flexibilización laboral y/o buscar mecanismos de socialización específicos para trabajadores transitorios.

\section{Estrategias relativas al producto}

La producción de bienes y servicios puede asumir tres opciones estratégicas: desarrollo de productos, diversificación o concentración. El desarrollo de productos surge por innovación de productos o mejora de los existentes; esta alternativa está relativamente restringida a empresas con capacidad para invertir en investigación y desarrollo de productos $(I \& D)$, en función de adquirir ventajas competitivas y mantener la vanguardia en el mercado, o responder a necesidades planteadas por la realidad; pero también es una opción para aquellas que mantienen incentivos a la creatividad de sus trabajadores.

La diversificación de las líneas de producción (Porter, 1999:129), consiste en incorporar productos desarrollados por otras empresas, para incursionar en nuevos mercados; esta estrategia implica legalmente gastos por concepto de patente o marca de fábrica, y eventualmente la adquisición de otras empresas con una línea diferente de producción, cuestión que nos traslada a una estrategia entre empresas.

La concentración radica en el reforzamiento de una línea de producción con énfasis en determinadas caracter ísticas del producto y centra la atención en un nicho del mercado actual (segmento con características particulares), al cual es dirigido ese producto. A continuación detallaremos las características básicas de las tres opciones.

\subsection{Desarrollo de productos}

El desarrollo de productos según Lambin (1995:343) consiste en aumentar las ventas desarrollando productos nuevos o mejorados dirigidos a los mercados actuales; aunque Kotler (1996:77) concibe esta estrategia solo para el desarrollo de nuevos productos de interés potencial. La perspectiva del desarrollo de productos es aumentar la participación 
en el mercado actual y generar nuevas oportunidades.

La creación de nuevos productos es un resultado directo de las actividades de Investigación y Desarrollo, lo mismo que la mejora de los existentes introduciendo variaciones, que permitan alterar su ciclo de vida y reposicionarlos en el mercado. Gorz (1977:274) plantea que el lanzamiento de productos mejorados pretende contrarrestar la tendencia a la baja en la tasa de ganancia por saturación del mercado, buscando incrementar la demanda para maximizar los beneficios.

Las oportunidades de mejora (Abell, 1995:28) están condicionadas por el tipo de mercancía; en productos relativamente sencillos de consumo masivo, las posibilidades parecen escasas, por la facilidad de que sean copiados por los competidores. Los productores centran su atención en el mejoramiento de productos existentes o en el desarrollo de enfoques realmente novedosos, con lo cual la innovación partiría de "una concepción mental sobre un nuevo modo de satisfacer al cliente (innovación conceptual)"; es decir, se realizaría no sólo a partir de las actividades de investigación y desarrollo con criterios técnicos y de ingeniería, sino con base en la concepción de los consumidores y del personal de la empresa, los cuales pueden realizar aportes creativos para nuevas soluciones. La evaluación determina los cambios que demanda el producto para responder a esos requerimientos y garantizar su diferenciación.

Las mejoras de producto están relacionadas directamente con dos tipos de cambio: sustanciales, que alteran su función esencial; y de apariencia, que representan modificaciones de estilo, en cuanto a características del producto (color, oportunidad, confort) y de presentación en cuanto a su empaque (Gorz, 1977:272). Sin embargo, según Stan y Meyer (1999:30) algunas “mejoras" tienden a acelerar el desgaste del producto, haciendo cada vez más cortos sus ciclos de vida, exigiendo sustituciones continuas para garantizar ventas futuras

\subsection{Diversificación productiva}

La diversificación comprende la adición de productos complementarios a las íneas actuales de producción, para entregar uno o más productos novedosos, que tengan cierta correspondencia con componentes tecnológicos y de mercado del producto actual; aunque la diversificación conglomerada planteada por David (1997:60), concibe la inclusión de nuevos negocios no relacionados con la actividad actual. La diversificación busca oportunidades de ingreso a segmentos de mercado con mejores perspectivas; pretende evitar la dependencia de actividades relacionadas con un solo negocio (Semler, 1999: 27; 
Lambin, 1995:343; Kotler, 1996:77).

En contraste con los autores mencionados, David (1997:59) afirma que las organizaciones tienen cada vez más problemas para administrar las actividades de diversos negocios, que fue la tendencia en los años 60 y 70, buscando no depender de una sola industria; pero en la actualidad la diversificación estaría en retirada. Sin embargo, sólo el análisis del tipo de negocio y sus condiciones actuales (demanda, proyecciones futuras, entorno) junto a la evaluación de los productos complementarios y no relacionados, podrá sugerir la pertinencia de optar por la diversificación. De hecho, en el mercado se observan experiencias de diversificación.

Para Semler (1999:27), cuando una empresa analiza los efectos que una recesión económica puede causar, o las restricciones del mercado en el cual opera, debe tener entre sus planes la alternativa de diversificación, antes que la crisis comience a afectarla.

\subsection{Concentración productiva}

Kotler (1996:84) y Lambin (1995: 339) presentan una concepción estratégica denominada "enfoque", que consiste en concentrar el negocio en uno o más segmentos estrechos del mercado, sin tratar de pugnar por participar en todo el mercado. El propósito es definir una población objetivo restringida y satisfacer las necesidades propias de ese segmento en mejores condiciones que los competidores, los cuales se dirigen a la totalidad del mercado. La concentración requiere orientar la producción bien sea hacia un segmento de amplia demanda, o al segmento estrecho pero de altos ingresos, que puede ser cubierto eficazmente en comparación con otras marcas.

La concentración se plantea como una estrategia para fortalecer la capacidad distintiva básica de la organización, centralizando los recursos en aquella actividad que represente una ventaja comparativa frente a los competidores. Sin embargo, el segmento de la industria seleccionado debe tener un amplio mercado, con potencial para crecer, que no sea crucial para el éxito de otros competidores importantes.

A diferencia de las anteriores una especie de concentración defensiva es la que David (1997:61, 69) denomina "encogimiento", cuando una organización se reagrupa buscando reducir costos y activos para revertir la caída de las ventas y utilidades; esta estrategia es reactiva, porque se establece en un ambiente de recursos limitados y presiones de los accionistas, empleados y de otros factores del entorno. 
La concentración productiva, conduce necesariamente a eliminar algunos procesos y, por consiguiente, los trabajadores involucrados, mediante despido o facilitando su conversión en empresarios.

\section{Conclusiones}

Para la clasificación de las estrategias intraempresa se asumió como criterio una concepción sistémica de proceso, factores de producción y resultados. De allí la identificación de los tres grandes tipos de estrategias: modernización de procesos, estrategias sobre la fuerza de trabajo y estrategias relativas al producto, vinculadas en principio a fines de participación en el mercado, reducción de costos, optimización de procesos y control de la fuerza de trabajo. Las estrategias intraempresa son de carácter competitivo, porque ninguna empresa decide invertir en mejoras internas, si no es con el fin de perfeccionar su capacidad para competir en el mercado.

La complejidad de los procesos internos hace que una sola estrategia intraempresa sea insuficiente para garantizar el éxito de la organización. Generalmente es necesario combinar estrategias, excepto en el caso de aquellas intrínsicamente opuestas, como la concentración productiva y la diversificación.

Las estrategias del mundo globalizado igual que las del pasado, se inscriben en la lógica del capital, con el propósito de incrementar la productividad, reducir los tiempos de respuesta 'mejorar' el producto o sustituirlo y reducir costos, todo ello para optimizar la posición competitiva en el mercado y por lo tanto la rentabilidad. Con estos fines, las empresas modifican y racionalizan sus procesos de trabajo, en función de la aplicación de nuevas tecnologías, e introducen cambios en la toma de decisiones, el mando y el control, así como en el tipo y características de los bienes o servicios que producen. Sin embargo, es preciso asumir las estrategias de modernización con una visión más amplia, que considere las condiciones y necesidades del contexto.

Las estrategias intraempresa generan consecuencias en la fuerza laboral y en la sociedad: a) desplazamiento de personal por tecnología, por reestructuración o por concentración productiva; b) intensificación del trabajo y precarización de las condiciones laborales. El incremento y generalización del desempleo, inevitablemente reducen el mercado potencial de las empresas, por lo cual no es un problema exclusivo de los asalariados, pues a largo plazo restringe ventas futuras, es decir, limita la realización de la mercancía, indispensable para la materialización de la ganancia. 


\section{Notas}

* Este artículo constituye un avance de la segunda etapa del Programa Estrategias de Modernización Empresarial, financiado por el Consejo de Desarrollo Científico y Humanístico (CONDES).

\section{Referencias Bibliográficas}

1. Abell, Derek (1995), Estrategias Duales. Compañía Editorial Continental S.A. México.

2. Albizu, Eneka (1997), Flexibilidad Laboral y Gestión de los Recursos Humanos. España. Ariel Sociedad Económica.

3. Bridges, William (1994), Fortune Magazine. The Time Inc. pp.14-21.

4. Carreón, Juan (2001), Comercio Electrónico. Eje de la era Digital http://www.excelsior.com.mx/ 9811/981113/fin20.html Internet. Consulta Julio 8/2001.

5. Castells, Manuel (1998), La era de la información. Economía, Sociedad y Cultura. Vol. 1. La sociedad Red. Alianza Editorial. Madrid, España.

6. Coriat, Benjamin (1995), Pensar al Revés: Trabajo y Organización en la Empresa Japonesa. México. Editores, S.A. Siglo XXI.

7. David, Fred R. (1997), Conceptos de Administración Estratégica. Editorial Prentice-Hall Hispanoamericana, S.A. $5^{\circ}$ Edición, México.

8. Dombois, Rainer y Pries, Ludger (1993), Modernización Empresarial. Tendencias en América Latina y Europa. Ediciones Nueva Sociedad Colombia.

9. Ermida, Oscar (1999), Las Relaciones de Trabajo en el siglo XXI. III Congreso Regional de las Américas. Asociación Peruana de Relaciones de Trabajo (APERT). pp. 1732.

10. Gamboa Cáceres, Teresa; Arellano Rodríguez, Madelein y Nava Vásquez, Yuneska (2001), “Estrategias Empresariales: Aproximación a una Tipología". En: Revista Venezolana de Ciencias Sociales. Volumen 5. No. 2. UNERMB. Cabimas, Venezuela. Pág. 132-154. 
11. Gorz, André (1977), "Técnica, técnicos y lucha de clases". En: Crítica de la División del Trabajo, textos escogidos y presentados por André Gorz. Editorial Laia B, Barcelona, España.

12. Hasty, Ron y Reardon, James (1998), Gerencia de Ventas al Detal. Mc Garw Hill Interamericana, S.A. Bogotá. Colombia.

13. Harrison, Bennett (1997), La empresa que viene. Editorial Paidós Ibérica, S.A. Barcelona, Madrid

14. Iglesias, Enrique (1999), Informe del Banco Interamericano de Desarrollo (B.I.D.). P.14.

15. Kotler, Philip (1996), Dirección de Mercadotecnia. Prentice-Hall Hispanoamericana, S.A. México.

16. Lambin, Jean-Jacques (1995), Marketing Estratégico. Mc Graw Hill Interamericana, S.A. España.

17. Montero, Cecilia, Alburquerque Mario y Jaime Ensignia (1999), "Recolocando el Trabajo en el Debate". En: Trabajo y Empresa entre dos siglos. Editorial Nueva Sociedad Venezuela. P. 9-15.

18. Olivera Lozano, Guillermo (1997), “Participación Complementaria e Integrada de la Pequeña Industria". En: Revista Comercio Exterior. Vol. 47. Num. 9. Septiembre. México http://www.cepal.cl/publicaciones/DesarrolloProductivo/4/Icl1404/lcl1404.pdf I Internet. Consulta: Mayo 30/2001.

19. Porter, Michael (2000), Ser competitivo. Nuevas aportaciones y conclusiones. Editorial Deusto. España.

20. Ramos, Joseph (1997), “Impacto de la modernización tecnológica”. Revista Capítulos “Cambios estratégicos en las políticas industriales” Edición No. 51 . Sistema Económico Latinoamericano (SELA). Caracas. http://lanic.utexas.edu/ sela/ capitulos/rcap514.htm. Internet. Consulta Jul 8/01.

21. Rifkin, Jeremy (1996), El fin del trabajo. Nuevas Tecnologías contra puestos de trabajo: el nacimiento de una nueva era. Paidós, Barcelona, España. 
22. Sant Roz, José (2003), Bolívar y Chávez ¡Alerta que caminan...! Fuerza Bolivariana de la Universidad de Los Andes. Mérida. Venezuela.

23. Semler, Ricardo (1999), Radical. El éxito de una empresa sorprendente. Editorial Gestión 2000. España.

24. Shumacher, E.F. (1990), Lo pequeño es hermoso. Apéndice de G. McRobie "Lo pequeño es posible" Herman Blume Ediciones. España.

25. Sierra Caballero, Francisco (1999), “Comunicación educativa y economía política. Apuntes sobre políticas culturales e innovación tecnológica". ÁMBITOS, Revista Andaluza de Comunicación No. 2. Enero-Junio. Universidad de La Laguna. Tenerife, Canarias. http://www.ull.es/ publicaciones/latina/aa2000vfe/sierra.html Internet. Consulta Mayo 30/2001.

26. Sotelo, Adrián (1999), Globalización y Precariedad del trabajo en México. Ediciones El Caballito, S.A. México.

27. Stan, Davis y Meyer, Christopher (1999), La velocidad de los cambios en la economía interconectada. Editorial Paidós. España.

28. Suárez Picón, Cira; Márquez, Y.; Nava, Y. y Ojeda, A. (2001), “Relaciones de Trabajo en el Contexto de la Globalización". En: Revista Venezolana de Gerencia, Año 6 № 15. Universidad del Zulia. Maracaibo, Venezuela.

29. Tapscott, Don (1997), La Economía Digital. Editorial Mc Graw Hill Interamericana, S.A. México.

30. Villalba, Julián (1996), Menú Estratégico. El arte de la Guerra Competitiva. Ediciones IESA. Venezuela.

31. Woolf, Brian (1995), Cómo adelgazar la cintura de su empresa. Cientos de ideas prácticas para reducir costos tomadas de los líderes en costos y ganancias. Panorama Editorial, México. 